\title{
Myoelectric manifestation of muscle fatigue in repetitive work detected by means of miniaturized sEMG sensors
}

\author{
Alberto Ranavolo, Giorgia Chini, Alessio Silvetti, Silvia Mari, Mariano Serrao \\ \& Francesco Draicchio
}

To cite this article: Alberto Ranavolo, Giorgia Chini, Alessio Silvetti, Silvia Mari, Mariano Serrao \& Francesco Draicchio (2017): Myoelectric manifestation of muscle fatigue in repetitive work detected by means of miniaturized sEMG sensors, International Journal of Occupational Safety and Ergonomics, DOI: 10.1080/10803548.2017.1357867

To link to this article: https://doi.org/10.1080/10803548.2017.1357867

\section{曲 Published online: 25 Sep 2017.}

Submit your article to this journal $\llbracket$

Џ Article views: 38

Q View related articles $₫$

View Crossmark data $\nearrow$ 


\title{
Myoelectric manifestation of muscle fatigue in repetitive work detected by means of miniaturized sEMG sensors
}

\author{
Alberto Ranavolo ${ }^{\mathrm{a}}$, Giorgia Chini ${ }^{\mathrm{b} *}$, Alessio Silvetti ${ }^{\mathrm{a}}$, Silvia Maric ${ }^{\mathrm{c}}$, Mariano Serrao ${ }^{\mathrm{c}, \mathrm{d}}$ and Francesco Draicchio ${ }^{\mathrm{a}}$ \\ ${ }^{a}$ Department of Occupational and Environmental Medicine, Epidemiology and Hygiene, INAIL, Italy; ${ }^{b}$ Department of Engineering, \\ Roma TRE University, Italy; ${ }^{c}$ Rehabilitation Centre Policlinico Italia, Italy; ${ }^{d}$ Department of Medical and Surgical Sciences and \\ Biotechnologies, Sapienza University of Rome, Italy
}

\begin{abstract}
Upper limb work-related musculoskeletal disorders have a 12-month prevalence ranging from 12 to $41 \%$ worldwide and can be partly caused by handling low loads at high frequency. The association between the myoelectric manifestation of elbow flexor muscle fatigue and occupational physical demand has never been investigated. It was hypothesized that an elbow flexor muscle fatigue index could be a valid risk indicator in handling low loads at high frequency. This study aims to measure the myoelectric manifestation of muscle fatigue of the three elbow flexor muscles during the execution of the work tasks in different risk conditions. Fifteen right-handed healthy adults were screened using a movement analysis laboratory consisting of optoelectronic, dynamometer and surface electromyographic systems. The main result indicates that the fatigue index calculated from the brachioradialis is sensitive to the interaction among risk classes, session and gender, and above all it is sensitive to the risk classes.
\end{abstract}

Keywords: repetitive work; myoelectric manifestation of muscle fatigue; upper limbs; ergonomics

\section{Introduction}

The recent development of microelectromechanical systems, such as inertial measurement units (IMUs) (i.e., combined accelerometers and gyroscopes) and surface electromyography (sEMG) sensors, has paved the way for some noteworthy scientific breakthroughs that may be applied to a range of research areas $[1,2]$ including human factors and ergonomics. In the field of manual handling, it is possible to define indices useful for prevention of workrelated musculoskeletal disorders (MSDs) that represent the most common disorders in occupational populations around the world [3]. Among these, upper limb workrelated musculoskeletal disorders (UL-WMSDs) have a 12 -month prevalence ranging from 12 to $41 \%$ worldwide [4]. UL-WMSDs can be partly caused by handling low loads at high frequency $[5,6]$, i.e., performing repetitive tasks [7].

To reduce the risk of UL-WMSDs, several methods have been developed to identify high-risk jobs that could be associated with an elevated risk of UL-WMSDs. Standard No. ISO 11228-3:2007 and part of Standard No. ISO/TR 12295:2014 [7,8] suggest the use of the occupational repetitive action (OCRA) checklist [9-11], the American Conference of Governmental Industrial
Hygienists' (ACGIH) assessment of hand activity level (HAL) $[12,13]$ and the strain index (SI) $[14,15]$. These methods are based on the ability to detect occupational physical risk factors and on the assessment of their physiological consequences. They allow risk classification using a multizone approach. The major limit of these methods is that, although providing quantitative outputs (table A.1 of Standard No. ISO 11228-3:2007 [7]), they require the assignment of scores to each risk determinant, such as work cycles, technical actions, repetitiveness, frequency of actions, forces, posture, movements and recovery times. Assigning a score to each determinant of risk for the criterion is not exactly and uniquely defined. The quantification of such scores is not easy, making these methods unrepeatable and unreliable [16-19]. These methods do not give emphasis to the role of physiological and biomechanical measures in the quantification of the risk process.

Repetitive work can lead to myoelectric manifestation of muscle fatigue [20-26], also called local muscle fatigue [27], which can be analyzed by another point of view. The myoelectric manifestation of muscle fatigue $[25,27,28]$ has received growing attention as a potential exposure metric in research toward prevention of UL-WMSDs at work. It is evaluated using bipolar and multichannel sEMG [29-31],

*Corresponding author. Email: giorgia.chini@gmail.com 
two objective and non-invasive physiological approaches widely used in ergonomics both in the laboratory and at the workplace [25,32-35]. In particular, the myoelectric manifestation of muscle fatigue is measured by the estimation of the decrease in the fiber conduction velocity (CV) [36,37]. The CV reflects a scaling of the power density function toward lower frequencies and an increase in amplitude variables of the sEMG signals [38,39]. Myoelectric manifestation of shoulder muscle fatigue has been proposed as a potential risk indicator in the prevention of shoulder work-related MSDs. It has been used in studies to investigate the biomechanical exposition of younger and older groups $[40,41]$ and the fatigue development in work with and without pause [22]. Further studies have reported trends indicating manifestations of fatigue of upper limb muscles [41] and of the trapezius muscle in a simulation of assembly tasks $[20,21,23,24]$. However, there are no known studies that have quantified the intensity level of the tasks. On the contrary, more recent findings have shown pronounced signs of development of myoelectric manifestations of shoulder muscle fatigue in different light levels of repetitive work [26,42-44].

UL-WMSDs include complaints of the upper arms, elbows, forearms, wrists and hands $[4,45]$. With regard to the elbow muscle actions in manual handling of low loads in high-frequency activities, these muscles are involved in developing internal flexor and extensor moments that contribute to the required net joint moments and power development. The association between the myoelectric manifestation of elbow flexor muscle fatigue and occupational physical demand has never been investigated. It was hypothesized that an elbow flexor muscle fatigue index could be a valid risk indicator in manual handling of low loads in high-frequency activities. This could be helpful in expanding the knowledge on which standardized risk factor assessment methods are based, directly supporting them and improving UL-WMSD prevention interventions using risk assessment approaches that accurately identify jobs at increased risk for injury. Optimization of the temporal aspects of repetitive work design requires a better understanding of the development of muscle fatigue in the elbow region over time.

The main objectives of this study were: to measure the myoelectric manifestation of muscle fatigue of the three elbow flexor muscles before and after four levels of simulated manual handling of low loads at high frequency performed and controlled under laboratory conditions; to analyze the calculated fatigue indices in order to understand whether they correctly classify the risk; to correlate calculated fatigue indices to the muscle behaviors during the execution of the dynamic work tasks (WTs).

The four levels were designed in such a way that the final scores could be classified by the OCRA checklist, ACGIH-HAL and SI methods as acceptable, very low, low and average risk conditions.

\section{Materials and methods}

\subsection{1 Subjects}

Fifteen right-handed healthy adults participated in the study (eight females, age $36 \pm 9$ years, range $25-42$ years, height $1.60 \pm 0.05 \mathrm{~m}$, weight $63 \pm 13 \mathrm{~kg}$; seven males, age $37 \pm 8$ years, range 26-44 years, height $1.73 \pm 0.09 \mathrm{~m}$, weight $79 \pm 15 \mathrm{~kg}$ ). The experiment was approved by the local ethics committee and complies with the Helsinki Declaration, and subjects signed a consent form prior to participating after a full explanation of the experimental procedure. All subjects recruited were non-athletes and led a sedentary lifestyle, did not practice physical activity in the gym and their jobs did not involve physical activity challenging the upper limbs. Subjects participating in any clinical drug trials, subjects suffering from any neurological disorders and with orthopedic disorders possibly causing trunk and upper limb impairment, such as scoliosis, back pain, hand deformities and painful musculoskeletal conditions, were excluded. No information regarding the expected results was provided in order to avoid bias, whether consciously or unconsciously.

\subsection{Instrumental evaluation}

All subjects recruited were screened using a movement analysis laboratory (see experimental procedures in Section 2.3) consisting of an optoelectronic motion analysis system used to detect movement during the execution of the WTs; a dynamometer mounted within a wooden structure used to measure the upward-directed force exerted by subjects during the fatiguing contractions; a multichannel sEMG apparatus used for fatigue estimation; a 16-channel Wi-Fi transmission sEMG to detect the sEMG behavior of the elbow flexor muscles during execution of the WTs.

\subsubsection{Optoelectronic recordings}

A SMART-E optoelectronic motion analysis system (BTS, Italy) was used. This consisted of eight infrared ray cameras (120-Hz sampling rate) to detect the motion of spherical markers covered with an aluminum powder reflecting material placed over prominent bony landmarks according to a simplified version of Rab et al. [46], and from the International Society of Biomechanics (ISB) recommendations $[47,48]$. Markers were positioned over the left and right acromion, the spinous process of the seventh cervical vertebra, the right olecranon, the right styloid process of the ulna and the left and right posterior superior iliac spine. Anthropometric data were collected for each subject. A calibration procedure was executed before the first data capture was performed. Three orthogonal marked axes were placed at the center of the laboratory and calibrated for approximately $5 \mathrm{~s}$ so as to define a global reference coordinate system. The movement of a marked wand, 


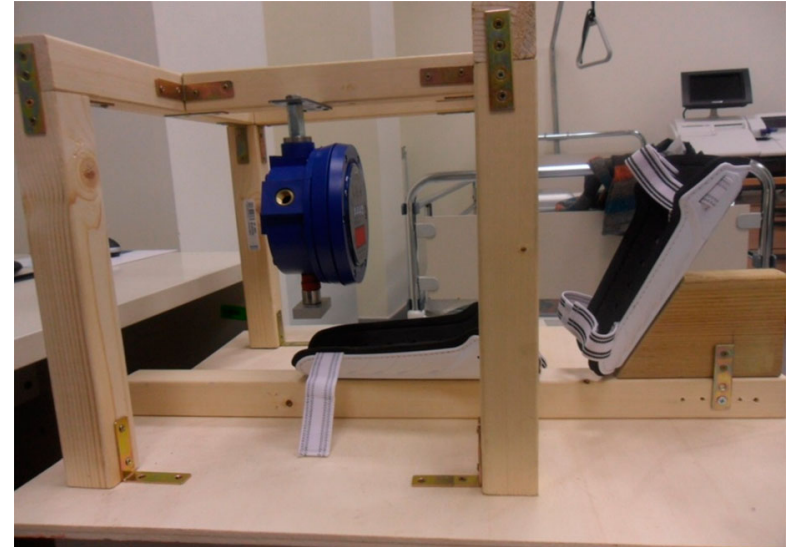

Figure 1. Wooden structure used to measure the upward-directed force exerted by subjects during the fatiguing contractions.

covering the work volume, was also captured for approximately $1 \mathrm{~min}$. Spatial accuracy was $<0.2 \mathrm{~mm}$ in the $x, y$ and $z$ dimensions.

\subsubsection{Dynamometer recordings and wooden structure}

A dynamometer (DIN ERGO 81-08 PRO X; Centro Italiano di Ergonomia, Italy) recorded the force and provided an instantaneous visual feedback of the force values through a display. The sample frequency was $5 \mathrm{~Hz}$. The dynamometer was fixed within a wooden structure (Figure 1) designed to measure the three elbow flexor muscles' myoelectric manifestation of fatigue.

\subsubsection{Multichannel sEMG recordings}

A 128-channel sEMG apparatus (EMG-USB128; LISiNOT Bioelettronica, Italy) was used during isometric contractions to detect muscle innervation zones (IZ) and tendon regions (TR) and to estimate the myoelectric manifestation of fatigue on the right biceps brachii caput longus (BL), biceps brachii caput brevis (BB) and brachioradialis (BR) muscles. An array of 16 dry electrodes placed sequentially along the muscle fiber direction over the $\mathrm{BL}$, $\mathrm{BB}$ and BR to detect the IZ and TR and three adhesive arrays of eight wet electrodes (LISiN; Spes Medica, Italy) placed between the detected IZ and TR were used to estimate the myoelectric manifestation of fatigue in these muscles. Electrodes of both dry and wet arrays had $5 \mathrm{~mm}$ in interelectrode distance and were used in a single differential configuration. The multichannel sEMG apparatus was synchronized with the dynamometer.

\subsubsection{Bipolar sEMG recordings}

Bipolar sEMG was acquired during dynamic contractions with a sampling rate of $1000 \mathrm{~Hz}$, using a 16-channel WiFi transmission surface electromyograph (FreeEMG300
System; BTS, Italy). A pre-process filtering and denoising procedure was performed. The lower and upper cut-off frequencies of the Hamming filter were 10 and $400 \mathrm{~Hz}$, respectively, and the common mode reaction ratio was $100 \mathrm{~dB}$. After skin preparation, bipolar $\mathrm{Ag} / \mathrm{AgCl}$ surface electrodes (H124SG; Kendall ARBO, Germany) prepared with electroconductive gel (diameter $1 \mathrm{~cm}$, distance between the electrodes $2 \mathrm{~cm}$ ) were placed along the direction of the muscle fibers of the BL, BB and BR according to the atlas of muscle IZ [49] and the location of the detected IZ and TR. Data from the surface electromyograph and the optoelectronic cameras were integrated and synchronized.

\subsection{Experimental procedures}

\subsubsection{IZ and TR detection}

Each subject was seen at the laboratory for 5 consecutive days (see Figure 2). On the first day, subjects did the IZ and TR detection and underwent theoretical and practical training to learn the experimental procedures to be done in the next 4 days. A dry array was placed sequentially over the BL, BB and BR while each subject was seated on a chair with the right arm placed within the wooden structure (Figure 1), the elbow joint at $110^{\circ}$ and the wrist supine in contact with the dynamometer. These tasks required the subject to exert an upward-directed force with the wrist activating the three elbow flexor muscles. The output of each electrode pair recorded the difference between the potentials detected between two electrodes. The electrode pair located near the IZ records a null signal while electrode pairs located on the opposite sides of the IZ record inverted voltage motor unit action potentials. These areas were marked with indelible ink so as to remain visible for the next 4 days of the experiment.

\subsubsection{Experimental sessions}

On each of the 4 days (see Figure 2) the subjects performed the isometric maximal voluntary contraction (iMVC) and the WT as described in the following two sub-sections.

2.3.2.1. Isometric maximal voluntary contractions. Each subject performed the iMVC using the wooden structure (fatiguing machine), to determine the maximum force using the dynamometer and the maximum sEMG value for amplitude normalization. These tasks were repeated three times after a rest period of $3 \mathrm{~min}$ between trials [30]. Three wet arrays were placed, between the IZ and the TR, along the direction of the muscle fiber direction of the BL, $\mathrm{BB}$ and $\mathrm{BR}$ of each subject who was seated on a chair with the right arm placed within the wooden structure, the elbow joint at $110^{\circ}$ and the wrist supine in contact with the dynamometer (Figure 1). These tasks required the subject 


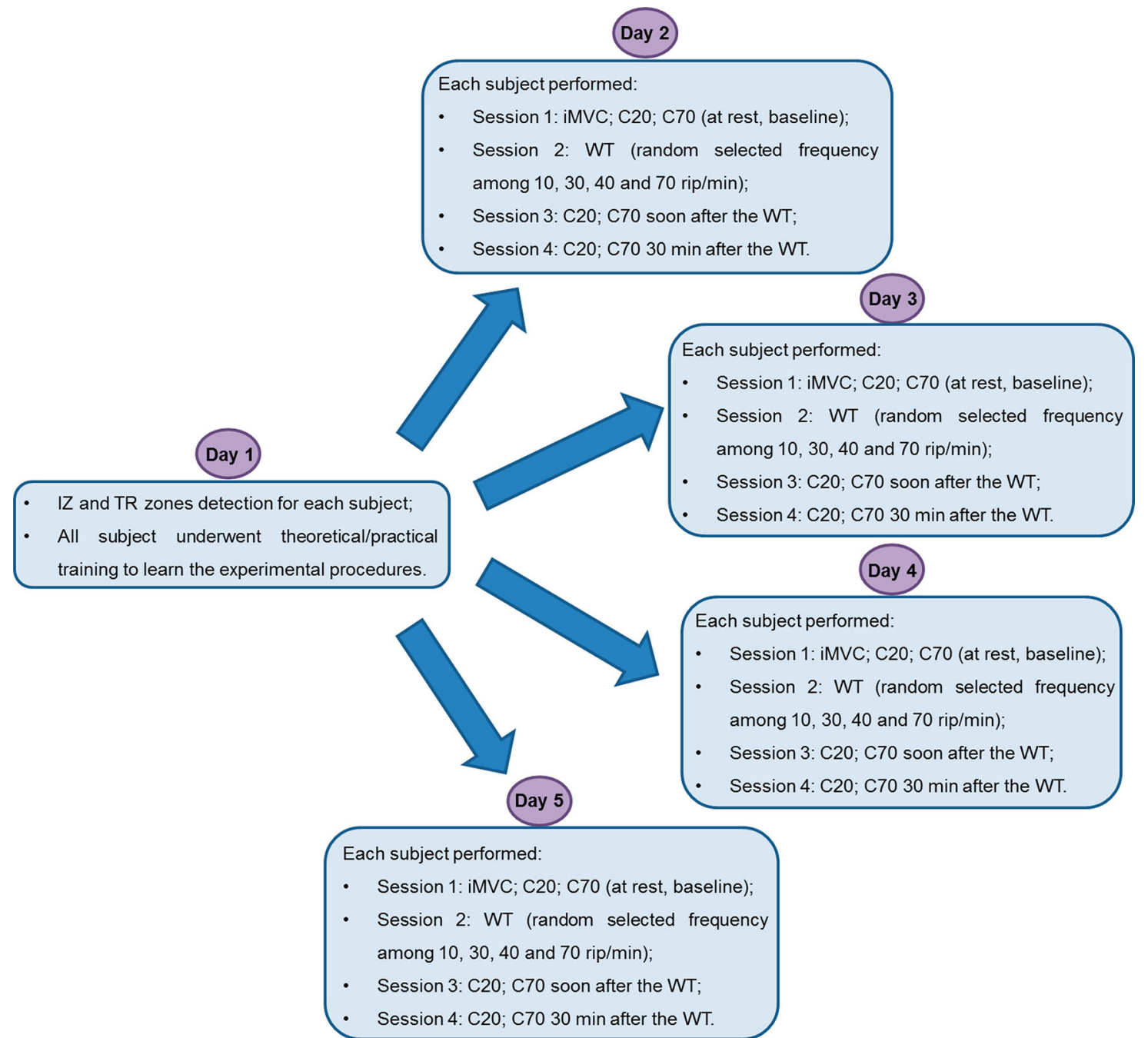

Figure 2. All stages of the experiment and explanation of what the subjects performed each day.

Note: C20 = isometric voluntary contraction at $20 \%$ of the maximum; C70 = isometric voluntary contraction at $70 \%$ of the maximum; $\mathrm{iMVC}=$ isometric maximal voluntary contraction; $\mathrm{IZ}=$ innervation zone; $\mathrm{TR}=$ tendon region; $\mathrm{WT}=$ work task.

to exert an upward-directed force with the wrist activating the elbow flexor muscles. To determine the maximal voluntary contraction (MVC) force, subjects were instructed to increase the force from zero to maximum and to hold it for $5 \mathrm{~s}$. After a resting period of $5 \mathrm{~min}$ a $20 \%$ iMVC contraction (C20) followed, and after a 3-min rest a 70\% iMVC contraction (C70) was performed. Subjects exerted a force target level of 20 and $70 \%$ of iMVC shown on the display of the dynamometer. The subjects were encouraged to keep these force levels as long as they could sustain an isometric contraction with the elbow flexor muscles, but never for more than $2 \mathrm{~min}$. The contractions were terminated when the subject deviated from the sub-maximal force for more than $3 \mathrm{~s}$.

The iMVCs at C20 and C70 were performed before (session 1) the WT (session 2), immediately after (session 3) and 30 min after (session 4) the WT (sessions 1, 3 and 4 were performed to acquire signals for fatigue estimation) (see Figure 2).
2.3.2.2. Work task. The WT consists of 'manual handling of low loads at high frequency' within the calibrated laboratory volume. During the WT we acquired the kinematics and bipolar sEMG in order to check the accuracy of the movement, in terms of frequencies and range of motions, and to analyze the behavior of the elbow flexor muscles. Before the execution of this task, the wet array of electrodes was removed and the bipolar surface electrodes were placed, between the IZ and TR, along the direction of the muscle fibers of the BL, BB and BR. Subjects performed four WTs (one WT per day, as shown in Figure 2) designed in such a way that the final scores can be classified using the OCRA checklist, ACGIH-HAL and SI methods as acceptable (AC), very low (VL), low (L) and average (AV) risk conditions (RC) (Tables 1 and 2). WT differed by varying the frequency of the actions: 10 (AC risk condition: WT10), 30 (VL risk condition: WT30), 40 (L risk condition: WT40) and 70 (AV risk condition: WT70) actions per minute. The frequency of action was 
Table 1. Assigned values for each risk factor assessed by HAL and SI methods.

\begin{tabular}{|c|c|c|c|c|c|c|c|c|c|}
\hline \multirow[b]{2}{*}{ Task } & \multicolumn{2}{|c|}{ Hand activity level } & \multicolumn{7}{|c|}{ Strain index } \\
\hline & Peak force & Activity level & Intensity & Duration & Efforts/minute & Posture & Speed & Duration per day & Score \\
\hline $10 \mathrm{rip} / \mathrm{min}$ & 3 & 1 & 3 & 0.5 & 1.5 & 1.0 & 1.0 & 0.5 & 2.250 \\
\hline $30 \mathrm{rip} / \mathrm{min}$ & 3 & 5 & 3 & 1.5 & 3.0 & 1.0 & 1.0 & 0.5 & 6.750 \\
\hline $40 \mathrm{rip} / \mathrm{min}$ & 3 & 5 & 3 & 1.5 & 3.0 & 1.0 & 1.5 & 0.5 & 10.125 \\
\hline $70 \mathrm{rip} / \mathrm{min}$ & 3 & 7 & 3 & 2.0 & 3.0 & 1.0 & 2.0 & 0.5 & 18.000 \\
\hline
\end{tabular}

Note: For HAL are indicated $x$ (peak force) and $y$ (activity level) values of the Cartesian coordinate system. $\mathrm{HAL}=$ hand activity level; $\mathrm{SI}=$ strain index.

Table 2. Assigned values for each risk factor assessed by the OCRA checklist.

\begin{tabular}{lcccccccr}
\hline & \multicolumn{7}{c}{ OCRA } \\
\cline { 2 - 9 } Task & Frequency & Force & Postures & Stereotypy & Additional & Duration & Recovery & Score \\
\hline $10 \mathrm{rip} / \mathrm{min}$ & 0 & 1 & 8 & 0.0 & 2 & 0.5 & 1 & 6.50 \\
$30 \mathrm{rip} / \mathrm{min}$ & 4 & 4 & 8 & 1.5 & 2 & 0.5 & 1 & 9.75 \\
$40 \mathrm{rip} / \mathrm{min}$ & 6 & 6 & 8 & 1.5 & 2 & 0.5 & 1 & 11.75 \\
$70 \mathrm{rip} / \mathrm{min}$ & 10 & 8 & 8 & 3.0 & 2 & 0.5 & 1 & 15.50 \\
\hline
\end{tabular}

Note: OCRA = occupational repetitive action.

designated by a digital auditory metronome while the order of the four experimental sessions was assigned randomly. High-risk zones were not taken into account in order to avoid real risks for the enrolled subjects. The WT consisted of a 10-min exercise, considered to be executed within a 1-h work duration. The subjects were seated, with the arm straight alongside the trunk (shoulder flexion of $0^{\circ}$ ) to limit the involvement of the shoulder, the back against the chair to minimize the activity of the erector spinae muscles and the feet raised to avoid contributions from the lower limb muscles. Participants were evaluated while performing right elbow flexion-extensions, with the elbow moving between $90^{\circ}$ and $135^{\circ}\left(0^{\circ}\right.$ indicates that the elbow is completely extended), through repeated handling of a dumbbell of $1 \mathrm{~kg}$ (hook grip hand pinch) grasped with the right hand. A vertical bar, with two references corresponding to the lower and upper limits for the wrist, helped the subjects to perform the desired movement amplitude.

\subsection{Data analysis}

C20 and C70 were processed using MATLAB version 7.0.4. For each muscle, three sEMG adjacent channels with a correlation coefficient signal higher than 0.75 were selected for further analysis.

With regard to the WT, kinematic and sEMG signals were acquired using Smart Capture version 1.10.462.0. After a tracking procedure (Smart Tracker version 1.10.462.0), which was required to assign a label to each spherical marker, data were processed using Smart Analyzer version 1.10.462.0 and MATLAB version 7.0.4. We excluded subjects whose mean angular displacement, measured by the optoelectronic motion analysis system, did not fall between $90^{\circ}$ and $135^{\circ}$ (with an error of $5 \%$ ) and whose frequency was different from what was recommended.

The mean of the three MVC forces was taken as the MVC force and used as a reference to calculate the target force for the fatiguing contractions.

\subsubsection{Fatigue parameters}

During the fatiguing contractions, MVC force and endurance at 20 and $70 \%$ of the MVC force (END20 and END70, respectively) were calculated. Endurance was defined as the time in which the subject was able to maintain the target force level. Fatigue plots were calculated on: muscle fiber CV (values were accepted if the average correlation coefficients between adjacent channels were higher than 0.75); average rectified value (ARV); root mean square (rms); mean frequency of the power spectrum (MNF); median frequency of the power spectrum (MDF). Fatigue indices were calculated for each signal by subdividing the signal into 500-ms epochs, within which amplitude and frequency parameters were computed. Because these parameters have proven to have a linear trend [28], the linear regression was evaluated (Figure 3A) and then data were normalized with respect to the intercept with the $y$ axis (Figure 3B). The normalized slope, indicating the variation rate with respect to the initial value, was used as the fatigue index.

\subsubsection{Work task parameters}

Kinematic and electromyographic data acquired during the execution of the WT were time normalized to the cycle duration and reduced to 100 samples using a polynomial 

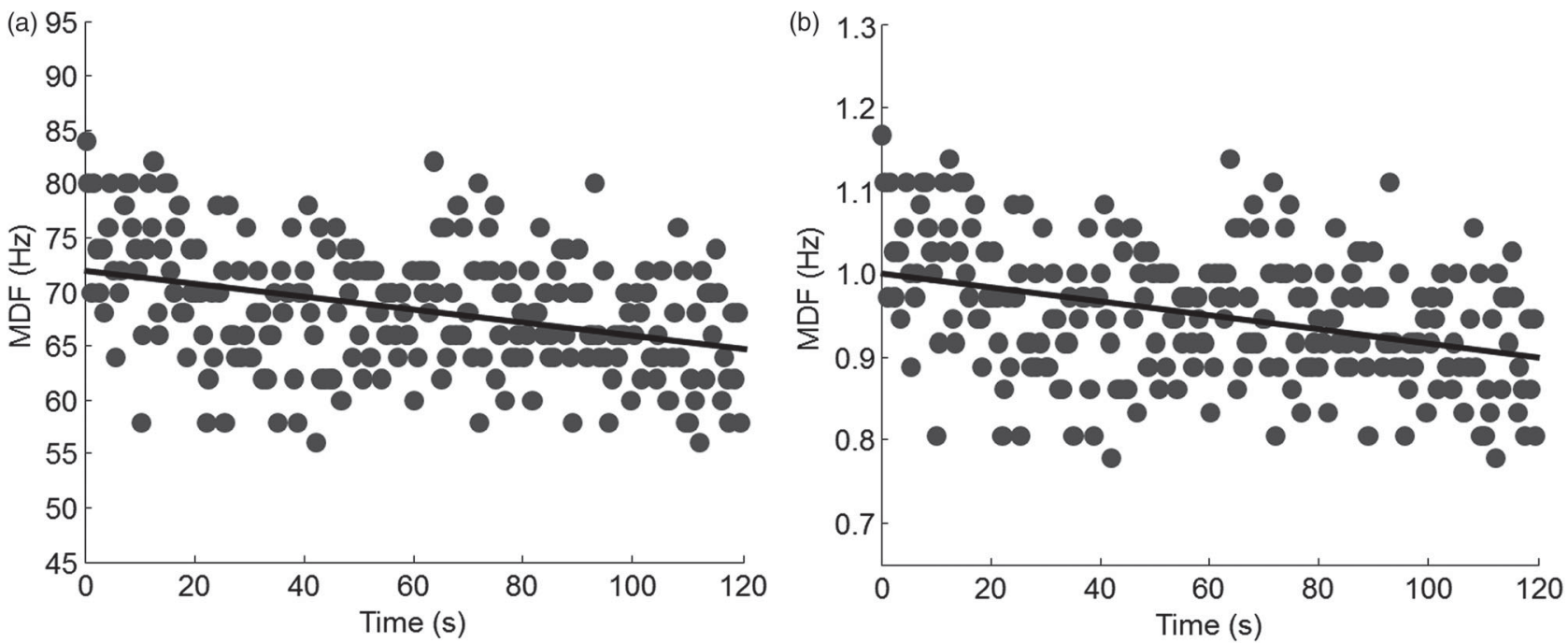

Figure 3. (a) MDF scatter plot with regression line and (b) MDF normalized scatter plot with normalized regression line for a representative subject and session.

Note: $\mathrm{MDF}=$ median frequency.

procedure. Each cycle was defined as the time period between two consecutive minimums of the vertical component of the marker positioned on the right styloid process of the ulna. Before the time normalization was performed, sEMG raw signals acquired during the MVC and WT were full-wave rectified with respect to the mean value and lowpass filtered using a 5-Hz Hamming filter, which yielded the linear envelopes for each sEMG signal. The rectified and filtered sEMG signals were expressed as a percentage of the sEMG peak value calculated as the mean of the maximum values detected for each of the three MVCs.

To characterize differences in timing and duration of sEMG, we calculated muscle activation timings (sum of the time periods in which the EMG activity exceeded 10\% of its maximum over each wrist cycle) and the full width at half maximum (FWHM, sum of the time periods in which the EMG signal overtakes half of its maximum over each wrist cycle). Analysis of the FWHM allowed us to quantify the duration of activity that tends to be the maximum for each muscle.

\subsection{Statistical analysis}

Statistical analysis was performed using PASW version 17 (PASW Statistic, formerly SPSS, USA). The ShapiroWilk test was applied to verify the null hypothesis that the acquired sample (in relation to the parameters calculated) came from a normally distributed population. A two-way analysis of variance (repeated-measures ANOVA) was performed to investigate the effects of the factors as well as their interactions on variables. We considered the risk class (RC; four levels: AC, VL, L, AV) and sessions (three levels: pre, post, post30) as within-subject factors and gender (two levels: male, female) as a between-subjects factor. Parametric paired $t$ tests were performed to detect any significant differences between conditions. $p<0.05$ was considered statistically significant. Bonferroni correction was used when multiple comparisons were performed.

Moreover, a one-way analysis of variance (repeatedmeasures ANOVA) was performed on the activation timing and on the FWHM. We considered the muscle (three levels: $\mathrm{BR}, \mathrm{BL}, \mathrm{BB})$ and the risk class $(\mathrm{RC}$; four levels: $\mathrm{AC}, \mathrm{VL}, \mathrm{L}, \mathrm{AV})$ as within-subject factors.

Holm-Sidak tests were performed to detect any significant differences between conditions.

\section{Results}

\subsection{Fatigue parameters}

All of the fatigue index values, calculated using ARV, rms, MNF and MDF, are reported in Table 3 for each muscle and risk condition.

\subsubsection{Maximal voluntary contraction}

A significant effect of session $(p=0.022)$ and gender $(p<0.001)$ were found in MVC force values. Post-hoc analysis revealed a significant difference in MVC force values between the pre and post sessions $(21.968 \pm 7.59$ and $20.932 \pm 7.67$ respectively, $p=0.002$ ) and between male and female subjects $(28.542 \pm 4.09$ and $15.347 \pm 3.71$ respectively, $p=0.026$ ).

\subsubsection{Endurance}

A significant effect of $\mathrm{RC}$ and gender interaction $(p=0.024)$ and of session and gender interaction ( $p=0.003)$ were found in END70. Post-hoc analysis showed significant differences in END70 between male and female subjects within the AC risk class 
Table 3. Results of EMG fatigue assessment during the iMVC at C20 and C70 for each muscle.

\begin{tabular}{|c|c|c|c|c|c|c|c|c|c|c|c|c|c|c|}
\hline \multirow[b]{2}{*}{ Task } & \multirow[b]{2}{*}{ Index } & \multirow[b]{2}{*}{ Muscle } & \multicolumn{3}{|c|}{ Average } & \multicolumn{3}{|c|}{ Very low } & \multicolumn{3}{|c|}{ Low } & \multicolumn{3}{|c|}{ Acceptable } \\
\hline & & & Pre & Post & Post30 & Pre & Post & Post30 & Pre & Post & Post30 & Pre & Post & Post30 \\
\hline \multirow[t]{12}{*}{$\mathrm{C} 20$} & \multirow[t]{3}{*}{ ARV } & $\mathrm{BR}$ & $2.9 \pm 3.7$ & $2.7 \pm 3.6$ & $5.4 \pm 6.8$ & $2.6 \pm 3.1$ & $2.2 \pm 3.1$ & $3.8 \pm 7.1$ & $4.9 \pm 9.2$ & $4.2 \pm 7.5$ & $1.7 \pm 4.8$ & $1.9 \pm 4.3$ & $5.2 \pm 7.4$ & $3.1 \pm 4.8$ \\
\hline & & $\mathrm{BB}$ & $3.4 \pm 5.0$ & $3.6 \pm 4.9$ & $3.3 \pm 4.2$ & $2.3 \pm 5.0$ & $2.3 \pm 3.6$ & $3.4 \pm 4.1$ & $1.2 \pm 2.3$ & $3.1 \pm 9.5$ & $0.6 \pm 4.9$ & $1.7 \pm 1.9$ & $7.6 \pm 8.0$ & $3.3 \pm 3.7$ \\
\hline & & $\mathrm{BL}$ & $0.7 \pm 2.0$ & $1.9 \pm 2.1$ & $3.8 \pm 7.4$ & $1.5 \pm 5.8$ & $3.3 \pm 5.8$ & $1.8 \pm 6.6$ & $0.2 \pm 1.4$ & $3.2 \pm 9.2$ & $1.1 \pm 6.4$ & $2.6 \pm 3.2$ & $5.5 \pm 7.7$ & $1.0 \pm 4.6$ \\
\hline & \multirow[t]{3}{*}{$\mathrm{rms}$} & $\mathrm{BR}$ & $3.0 \pm 3.9$ & $3.1 \pm 3.8$ & $4.8 \pm 6.9$ & $2.8 \pm 3.2$ & $2.4 \pm 3.3$ & $3.7 \pm 6.8$ & $5.0 \pm 9.9$ & $4.4 \pm 7.8$ & $1.9 \pm 5.1$ & $1.9 \pm 3.9$ & $5.5 \pm 7.7$ & $3.2 \pm 4.2$ \\
\hline & & $\mathrm{BB}$ & $3.2 \pm 4.5$ & $3.5 \pm 4.7$ & $3.4 \pm 4.1$ & $2.2 \pm 4.8$ & $2.3 \pm 3.7$ & $3.3 \pm 3.9$ & $1.2 \pm 2.3$ & $3.0 \pm 9.1$ & $0.6 \pm 4.9$ & $1.8 \pm 1.9$ & $7.6 \pm 7.7$ & $3.3 \pm 3.6$ \\
\hline & & BL & $0.8 \pm 2.0$ & $1.9 \pm 2.1$ & $4.3 \pm$ & $1.4 \pm 3.4$ & $3.6 \pm 6.5$ & $2.1 \pm 7.1$ & $0.2 \pm 1.6$ & $3.4 \pm 9.8$ & & $2.7 \pm 3.3$ & $5.2 \pm 7.1$ & $1.1 \pm 4.5$ \\
\hline & \multirow[t]{3}{*}{ MDF } & $\mathrm{BR}$ & $-0.5 \pm 0.8$ & $-0.9 \pm 0.8$ & $-2.5 \pm 5.4$ & $-0.04 \pm 1.1$ & $-0.9 \pm 1.1$ & $-0.8 \pm 0.8$ & $-0.3 \pm 1.6$ & $-1.2 \pm 1.0$ & $-0.8 \pm 0.6$ & $-0.7 \pm 0.8$ & $-1.0 \pm 0.9$ & $-1.0 \pm 0.9$ \\
\hline & & $\mathrm{BB}$ & $0.4 \pm 1.0$ & $-0.09 \pm 0.04$ & $-0.5 \pm 1.4$ & $-0.4 \pm 0.6$ & $-0.6 \pm 0.8$ & $-1.0 \pm 1.6$ & $-0.2 \pm 0.5$ & $-0.5 \pm 0.4$ & $-0.5 \pm 0.7$ & $0.09 \pm 0.5$ & $-0.5 \pm 0.5$ & $-0.4 \pm 0.9$ \\
\hline & & $\mathrm{BL}$ & $2 \pm 1.5$ & $-0.5 \pm 0.5$ & $-1.5 \pm 4.1$ & $-0.2 \pm 0.6$ & $-0.8 \pm 0.6$ & $-0.4 \pm 0.3$ & $-0.3 \pm 0.4$ & $-0.4 \pm 1.2$ & $-0.7 \pm 0.7$ & $-0.2 \pm 0.4$ & $-0.9 \pm 1.2$ & $-0.2 \pm 1.8$ \\
\hline & \multirow[t]{3}{*}{ MNF } & $\mathrm{BR}$ & $-0.3 \pm 0.9$ & $-0.9 \pm 0.9$ & $-2.1 \pm 4.5$ & $-0.7 \pm 0.9$ & $-0.8 \pm 0.7$ & $-0.8 \pm 0.8$ & $-0.4 \pm 1.7$ & $-1.1 \pm 1.3$ & $-0.8 \pm 0.6$ & $-0.6 \pm 0.7$ & $-0.88 \pm 0.93$ & $-0.27 \pm 0.76$ \\
\hline & & $\mathrm{BB}$ & $1.1 \pm 7.4$ & $-0.2 \pm 4.2$ & $-0.6 \pm 1.2$ & $-0.4 \pm 0.8$ & $-0.6 \pm 0.9$ & $-0.8 \pm 1.3$ & $-0.2 \pm 0.4$ & $-0.3 \pm 0.7$ & $-0.3 \pm 0.6$ & $-0.6 \pm 0.4$ & $-0.40 \pm 0.78$ & $-0.40 \pm 0.78$ \\
\hline & & $\mathrm{BL}$ & $0.2 \pm 0.3$ & $-0.5 \pm 0.6$ & $-1.2 \pm 4.9$ & $-0.3 \pm 0.7$ & $-0.7 \pm 0.9$ & $-0.4 \pm 1.2$ & $-0.3 \pm 0.6$ & $-0.6 \pm 0.7$ & $-0.5 \pm 0.6$ & $-0.3 \pm 0.7$ & $-0.93 \pm 1.82$ & $-0.10 \pm 0.79$ \\
\hline \multirow[t]{12}{*}{$\mathrm{C} 70$} & \multirow[t]{3}{*}{ ARV } & $\mathrm{BR}$ & $9.1 \pm 10.6$ & $15.8 \pm 26$ & & $17.5 \pm 38.1$ & $20.8 \pm 58.5$ & $16.5 \pm 26.9$ & $9.6 \pm 15.8$ & $12.4 \pm 16.3$ & & $7.2 \pm 7.5$ & $16.3 \pm 24.5$ & $14.3 \pm 12.8$ \\
\hline & & $\mathrm{BB}$ & $24.7 \pm$ & $35.1 \pm$ & & & & $12.9 \pm$ & $3.9=$ & $7.2 \pm 14.6$ & $2.9=$ & $3.3 \pm 11.4$ & $8.1=$ & $11.4 \pm$ \\
\hline & & BL & $11.2 \pm 22.8$ & $12.2 \pm 18.7$ & $1.9 \pm 1.3$ & $9.4 \pm 14.4$ & $5.1 \pm 16.8$ & $13.2 \pm 13.9$ & $4.2 \pm 10.9$ & $7.8 \pm 14.1$ & $0.5 \pm 15.1$ & $4.1 \pm 15.0$ & $8.7 \pm 9.7$ & $7.9 \pm 23.3$ \\
\hline & \multirow[t]{3}{*}{$\mathrm{rms}$} & $\mathrm{BR}$ & $9.1 \pm 9.9$ & $15.8 \pm 24.1$ & $6.4 \pm 11.5$ & $17.6 \pm 35.1$ & $22.2 \pm 61.7$ & $15.8 \pm 24.2$ & $9.5 \pm 15.5$ & $13.4 \pm 16.8$ & $6.6 \pm 9.3$ & $7.3 \pm 7.9$ & $16.2 \pm 23.8$ & $19.0 \pm 17.1$ \\
\hline & & $\mathrm{BB}$ & $24.1 \pm 54.5$ & $35.2 \pm 92.7$ & $2.7 \pm 7.5$ & $7.7 \pm 12.9$ & $4.9 \pm 9.5$ & $13.1 \pm 12.2$ & $5.5 \pm 11.0$ & $7.5 \pm 15.1$ & $3.6 \pm 9.5$ & $3.7 \pm 10.7$ & $8.1 \pm 6.7$ & $11.4 \pm 17.2$ \\
\hline & & $\mathrm{BL}$ & $11.2 \pm 22.2$ & $12.5 \pm 18.2$ & $2.2 \pm 12.3$ & $9.6 \pm 14.2$ & $5.6 \pm 16.4$ & $13.5 \pm 13.6$ & $3.4 \pm 10.7$ & $6.6 \pm 13.4$ & $2.2 \pm 14.7$ & $4.4 \pm 14.2$ & $8.7 \pm 9.5$ & $8.3 \pm 23.3$ \\
\hline & \multirow[t]{3}{*}{ MDF } & $\mathrm{BR}$ & $-7.1 \pm 3.8$ & $-6.9 \pm 3.2$ & $-6.6 \pm 3.2$ & $-6.0 \pm 2.6$ & $-5.5 \pm 3.2$ & $-7.5 \pm 3.2$ & $-7.3 \pm 3.8$ & $-5.7 \pm 2.9$ & $-7.7 \pm 4.8$ & $-7.5 \pm 4.8$ & $-4.8 \pm 4.3$ & $-7.6 \pm 5.5$ \\
\hline & & $\mathrm{BB}$ & $-7.6 \pm 5.1$ & $-9.4 \pm 6.2$ & $-6.1 \pm 2.3$ & $-7.5 \pm 3.3$ & $-6.7 \pm 3.1$ & $-7.8 \pm 2.7$ & $-9.5 \pm 3.1$ & $-7.9 \pm 4.1$ & $-7.6 \pm 3.5$ & $-7.7 \pm 5.3$ & $-5.8 \pm 4.1$ & $-10.9 \pm 7.7$ \\
\hline & & BL & $-6.8 \pm 3.1$ & $-5.6 \pm 6.6$ & $-6.4 \pm 3.1$ & $-6.4 \pm 2.9$ & $-5.3 \pm 2.9$ & $-5.8 \pm 2.5$ & $-6.7 \pm 4.3$ & $-4.7 \pm 3.1$ & $-5.9 \pm 2.0$ & $-6.5 \pm 4.1$ & $-6.9 \pm 3.4$ & $-6.9 \pm 4.4$ \\
\hline & \multirow[t]{3}{*}{ MNF } & $\mathrm{BR}$ & $-7.1 \pm 3.4$ & $-6.1 \pm 4.9$ & $-6.7 \pm 2.8$ & $-6.1 \pm 2.7$ & $-5.4 \pm 2.6$ & $-6.8 \pm 3.3$ & $-7.1 \pm 3.5$ & $-5.5 \pm 2.6$ & $-7.1 \pm 4.1$ & $-6.9 \pm 4.7$ & $-5.1 \pm 3.6$ & $-8.2 \pm 5.1$ \\
\hline & & $\mathrm{BB}$ & $-9.3 \pm 7.2$ & $-9.4 \pm 6.9$ & $-6.0 \pm 2.5$ & $-6.9 \pm 3.2$ & $-6.9 \pm 3.2$ & $-8.1 \pm 3.2$ & $-9.4 \pm 3.6$ & $-7.8 \pm 3.9$ & $-7.9 \pm 3.5$ & $-7.9 \pm 5.4$ & $-6.9 \pm 4.3$ & $-10.9 \pm 7.4$ \\
\hline & & $\mathrm{BL}$ & $-6.6 \pm 3.3$ & $-5.9 \pm 4.9$ & $-6.3 \pm 3.4$ & $-5.6 \pm 2.9$ & $-5.3 \pm 2.5$ & $-5.4 \pm 2.7$ & $-6.9 \pm 3.9$ & $-4.5 \pm 4.0$ & $-5.8 \pm 2.3$ & $-6.6 \pm 4.1$ & $-7.6 \pm 3.1$ & $-7.4 \pm 6.9$ \\
\hline
\end{tabular}

Note: $M \pm S D$ of each parameter reported for each risk condition (average, very low, low and acceptable) and session (before the working task, soon after the working task and 30 min after the working task, corresponding to pre, post and post30). All values must be multiplied by $10^{-3}$. ARV = average rectified value; $\mathrm{BB}=$ biceps brachii caput brevis; $\mathrm{BL}=$ biceps brachii caput longus; $\mathrm{BR}=$ brachioradialis; $\mathrm{C} 20=$ isometric voluntary contraction at $20 \%$ of the maximum; $\mathrm{C} 70=$ isometric voluntary contraction at $70 \%$ of the maximum; $\mathrm{EMG}=$ electromyographic; $\mathrm{iMVC}=$ isometric maximal voluntary contraction; $\mathrm{MDF}=$ median frequency; $\mathrm{MNF}=$ mean frequency; $\mathrm{rms}=$ root mean square. 


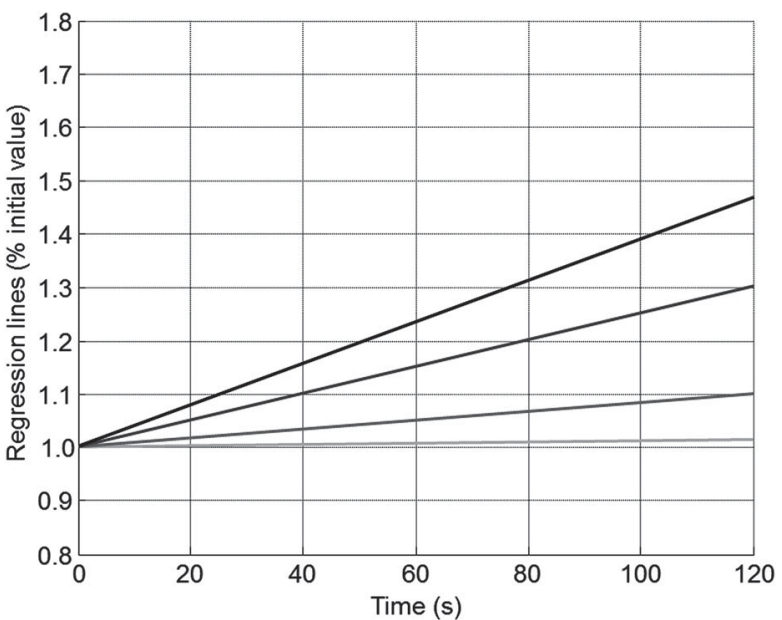

Figure 4. Increasing slopes of the regression lines for rms 70 of the brachioradialis muscle for a representative subject within risk classes.

Note: From the lightest gray corresponding to the acceptable risk class to the darkest gray corresponding to the average risk class. rms $70=$ root mean square of the isometric contraction at $70 \%$ of isometric maximal voluntary contraction.

$(140.40 \pm 42.57$ and $219.11 \pm 61.46$ respectively, $p=$ 0.001 ), between male and female subjects within the post30 session $(149.35 \pm 52.25$ and $221.91 \pm 80.44$ respectively, $p=0.001)$ and between pre and post30 sessions in male participants $(189.50 \pm 52.88$ and $149.35 \pm 52.25$ respectively, $p=0.004)$.

\subsubsection{Brachioradialis}

A significant effect of RC, gender and session interaction ( $p=0.038)$, of RC and gender interaction $(p=0.005)$ and of RC ( $p=0.049)$ was found in MNF20 and rms70 respectively. Figure 4 shows the increasing slope of the regression lines within risk classes. Post-hoc analysis showed significant differences in rms 70 between each pair of risk class except for AC vs. VL and AC vs. L (Figure 5).

\subsubsection{Biceps brachii caput longus}

The repeated measurement using ANOVA revealed a significant effect of gender on MNF20 $(p=0.038)$ and of session on MDF20 ( $p=0.004)$. When performing the post-hoc analysis on BL muscle parameters, a significant difference was found between pre and post in MDF20 $(-0.000116 \pm 0.000491$ and $0.000645 \pm 0.000932$ respectively, $p=0.002)$ and between males and females in MNF20 $(-0.000895 \pm 0.002285$ and $-0.0001780 \pm$ 0.0006334 respectively, $p=0.021$ ).

\subsubsection{Biceps brachii caput brevis}

The repeated measurement using ANOVA revealed a significant effect of session on MDF20 $(p=0.025)$ and MNF20 ( $p=0.037)$ and a significant effect of session

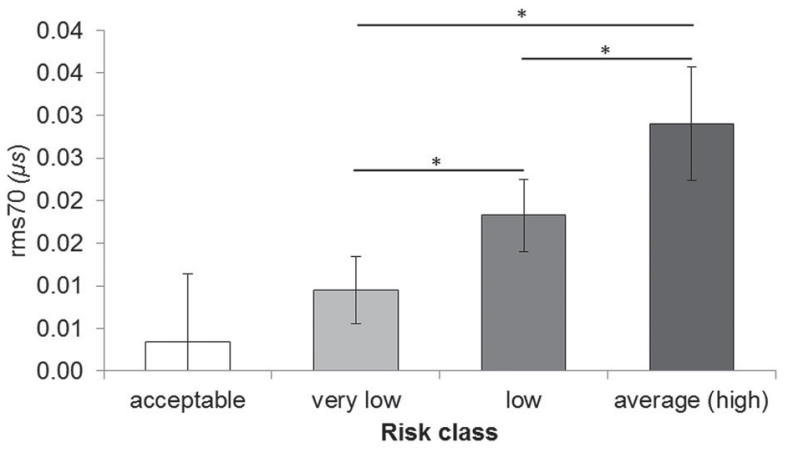

Figure 5. Mean (bar height) and standard deviation (error bar) rms70 in each risk class.

Note: Horizontal lines with asterisks denote a statistically significant difference between each pair of risk class except for acceptable vs. very low and acceptable vs. low. rms $70=$ root mean square of the isometric contraction at $70 \%$ of isometric maximal voluntary contraction.

and RC interaction on MDF70 ( $p=0.029)$. When investigating the effect of session, we observed significant differences in MDF20 between the sessions pre-post $(-0.0000110 \pm 0.0007340$ and $-0.0004360 \pm 0.0005823$ respectively, $p=0.002)$ and pre-post30 $(-0.0000110 \pm$ 0.0007340 and $-0.0006140 \pm 0.0012028$ respectively, $p=0.003$ ). Analogous results were obtained for MND20, for which significant differences were revealed between the sessions pre-post $(-0.0000680 \pm 0.0006857$ and $-0.0004090 \pm 0.0006809$ respectively, $p=0.011)$ and pre-post30 $(-0.0000680 \pm 0.0006857$ and $-0.0005320 \pm$ 0.0010039 respectively, $p=0.009$ ).

\subsection{Work task parameters}

\subsubsection{One-way ANOVA among muscles (at each risk class)}

Results for muscle activation timing are shown in Figure 6. There was no significant effect among muscles at the AC and VL levels. At the AV level, the one-way ANOVA

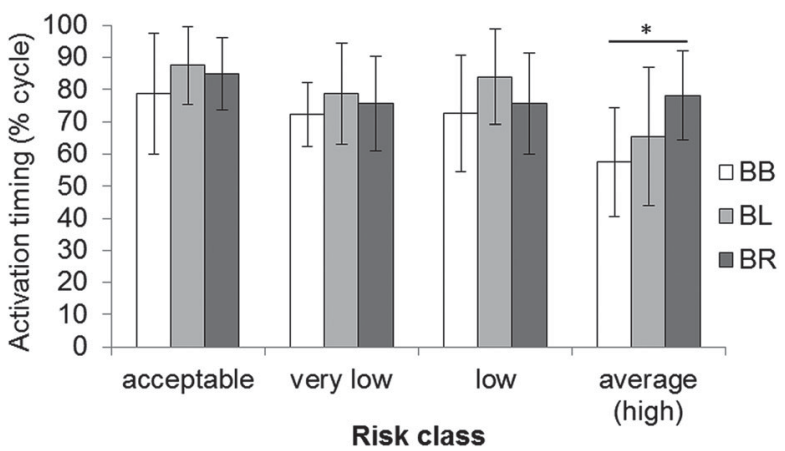

Figure 6. Mean (bar height) and standard deviation (error bar) for each muscle activation timing in each risk class.

Note: Horizontal line with asterisk denotes a statistically significant difference between BB and BR at average risk level. $\mathrm{BB}=$ biceps brachii caput brevis; $\mathrm{BL}=$ biceps brachii caput longus; $\mathrm{BR}=$ brachioradialis. 

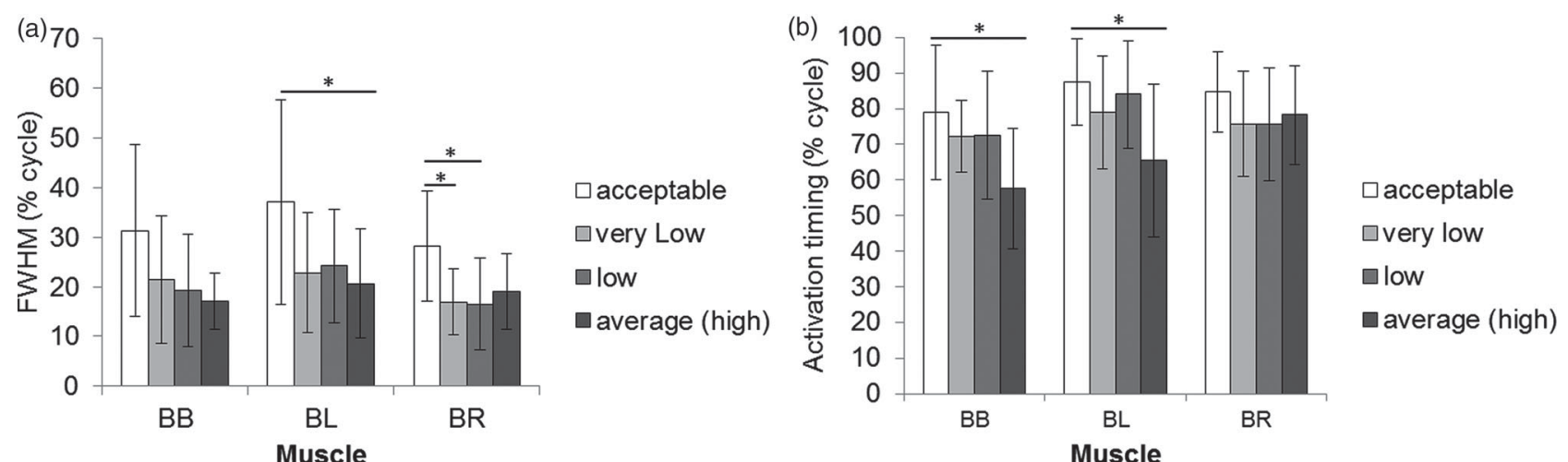

Figure 7. Mean (bar height) and standard deviation (error bar) of (a) the full width at half maximum and (b) the activation timing at each risk level for each muscle.

Note: Horizontal lines with asterisks denote a statistically significant difference in the activation timing and in the FWHM of the BL muscle between the acceptable and the average levels and in the activation timing of the BB muscle between the acceptable and the average levels. $\mathrm{BB}=$ biceps brachii caput brevis; $\mathrm{BL}=$ biceps brachii caput longus; $\mathrm{BR}=$ brachioradialis; $\mathrm{FWHM}=$ full width at half maximum.

revealed a significant main effect on the activation timing $(p=0.033)$. Post-hoc analysis showed significant differences in activation timing between $\mathrm{BR}$ and $\mathrm{BB}(p=0.01)$.

\subsubsection{One-way ANOVA among risk classes (for each muscle)}

Results for risk level are shown in Figure 7. With regard to $\mathrm{BR}$, the one-way ANOVA among RC revealed a significant main effect on the FWHM $(p=0.006)$. Post-hoc analysis showed significant differences in the FWHM of the BR muscle between the AC and the VL levels $(p=0.009)$ and between the AC and the L levels ( $p=0.015)$.

The one-way ANOVA of the BL revealed a significant main effect on the activation timing $(p=0.009)$ and on the FWHM ( $p=0.039)$. Post-hoc analysis showed significant differences in the activation timing and in the FWHM of the $\mathrm{BL}$ muscle between the AC and the AV levels ( $p=0.01$ and $p=0.049$ ).

\section{Discussion}

In this study we investigated for the first time the myoelectric manifestations of muscle fatigue of the three elbow muscle flexors before and after repetitive WTs which were later classified by the final score of the standardized risk assessment methods as acceptable (AC), very low (VL), low (L) and average (AV) risk conditions (RC). We also investigated the electrical behavior of these three muscles during the execution of the WT. In particular, we found that the fatigue index calculated from the $\mathrm{BR}$ is sensitive to the interaction among $\mathrm{RC}$, session and gender, and above all it is sensitive to the RC. In detail, it is interesting to emphasize that this fatigue index calculated using the rms at $70 \%$ of the MVC differs among the four RC investigated, except in the comparison of the AC vs. VL and the AC vs. L. This result suggests that the BR can be used in studies in which it is necessary to define the risk class for a WT and also to track the modification of this index following corrective ergonomic actions aimed at the reduction of the injury rates. This is especially true when the WT involves the substantial use of the elbow joint and when it is necessary to change the motion adaptation occurring to reduce the biomechanical load in the fatigued shoulder region while still maintaining task requirements [50]. The rms is recognized to be a good sEMG parameter in the construction of the myoelectric manifestation of muscle fatigue by other researchers who have also investigated repetitive work [20-22]. The analysis of the activation timings of the three elbow flexor muscles showed the increased myoelectric fatigability of BR derived from the prolonged activation, with respect to the $\mathrm{BL}$ and $\mathrm{BB}$ within the $\mathrm{AV}$ risk class.

When it is necessary to analyze differences in myoelectric manifestation of muscle fatigue before and after a work shift it is preferable to compute the fatigue index using the frequency parameters (MNF and MDF) at 20\% of the MVC in the BL. This is probably due to the fact that this percentage of MVC recruits the same muscle fibers in the execution of the WT during low levels of risk class. Limitations of the study are related to the sEMG method, and include the presence of crosstalk, noise and problems related to the electrode location, electrode-skin impedance, size, configuration and distance [51]. To minimize these problems, we used the European Recommendations for Surface Electromyography (SENIAM) [52] and the Atlas of Muscle Innervation Zones [49] as references.

\section{Conclusion}

In light of these considerations, we believe that changes in myoelectric manifestations of muscle fatigue measured on the BR could be used to identify different classes of riskrelated work activities characterized by handling of low 
loads at high frequency. This is especially true when it is of importance to investigate the efficacy of ergonomic intervention, such as the introduction of pauses in activities that do not already include them.

\section{Disclosure statement}

No potential conflict of interest was reported by the authors.

\section{References}

[1] Taborri J, Palermo E, Rossi S, et al. Gait partitioning methods: a systematic review. Sensors (Basel). 2016;16(1):66. Pii: E66. doi:10.3390/s16010066

[2] Sprager S, Juric MB. Inertial sensor-based gait recognition: a review. Sensors (Basel). 2015;15(9):22089-22127. doi:10.3390/s150922089

[3] Hoe VC, Urquhart DM, Kelsall HL, et al. Ergonomic design and training for preventing work-related musculoskeletal disorders of the upper limb and neck in adults. Cochrane Database Syst Rev. 2012;15(8):CD008570.

[4] Huisstede BMA, Bierma-Zeinstra SMA, Koes BW, et al. Incidence and prevalence of upper-extremity musculoskeletal disorders. A systematic appraisal of the literature. BMC Musculoskelet Disord. 2006;7:218. doi:10.1186/14712474-7-7

[5] Colombini D, Occhipinti E. Preventing upper limb work-related musculoskeletal disorders (UL-WMSDS): new approaches in job (re)design and current trends in standardization. Appl Ergon. 2006;37(4):441-450. doi:10.1016/j.apergo.2006.04.008

[6] Kim E-A, Nakata M. Work-related musculoskeletal disorders in Korea and Japan: a comparative description. Ann Occup Environ Med. 2014;26:1380. doi:10.1186/20524374-26-17

[7] International Organization for Standardization (ISO). Ergonomics - manual handling - part 3: handling of low loads at high frequency. Geneva: ISO; 2007. Standard No. ISO 11228-3:2007.

[8] International Organization for Standardization (ISO). Ergonomics - application document for International Standards on manual handling (ISO 11228-1, ISO 11228-2 and ISO 11228-3) and evaluation of static working postures (ISO 11226). Geneva: ISO; 2014. Standard No. ISO/TR 12295:2014.

[9] Occhipinti E. OCRA: a concise index for the assessment of exposure to repetitive movements of the upper limbs. Ergonomics. 1998;41(9):1290-1311. doi:10.1080/00140 1398186315

[10] Colombini D, Occhipinti E, Grieco A. La valutazione e la gestione del rischio da movimenti e sforzi ripetuti degli arti superiori. Analisi organizzative, indici di esposizione OCRA, schemi di intervento e principi di riprogettazione [Risk assessment and management of repetitive movements and exertions of upper limbs. Job analysis, OCRA risk index, prevention strategies and design principles]. Milan: Franco Angeli; 2002. Italian.

[11] Occhipinti E, Colombini D. The occupational repetitive action (OCRA) methods: OCRA index and OCRA checklist. In: Stanton NA, Hedge A, Brookhuis K, et al., editors. Handbook of human factors and ergonomics methods. New York (NY): CRC Press; 2004. p. 145-161.

[12] American Conference of Governmental Industrial Hygenists. Guide to occupational exposure values. Cleveland $(\mathrm{OH})$ : ACGIH; 2001.
[13] Radwin RG, Azari DP, Lindstrom MJ, et al. A frequencyduty cycle equation for the ACGIH hand activity level. Ergonomics. 2015;58(2):173-183. doi:10.1080/00140139. 2014.966154

[14] Steven Moore J, Garg A. The Strain Index: a proposed method to analyze jobs for risk of distal upper extremity disorders. Am Ind Hyg Assoc J. 1995;56(5):443-458. doi:10.1080/15428119591016863

[15] Garg A, Moore JS, Kapellusch JM. The Revised Strain Index: an improved upper extremity exposure assessment model. Ergonomics. 2016;14:1-11.

[16] Bao S, Spielholz P, Howard N, et al. Quantifying repetitive hand activity for epidemiological research on musculoskeletal disorders - part I: individual exposure assessment. Ergonomics. 2006;49(4):361-380. doi:10.1080/00140130 500520214

[17] Bao S, Howard N, Spielholz P, et al. Quantifying repetitive hand activity for epidemiological research on musculoskeletal disorders - part II: comparison of different methods of measuring force level and repetitiveness. Ergonomics. 2006;49(4):381-392. doi:10.1080/00140130600555938

[18] Spielholz P, Bao S, Howard N, et al. Reliability and validity assessment of the hand activity level threshold limit value and strain index using expert ratings of mono-task jobs. J Occup Environ Hyg. 2008;5(4):250-257. doi:10.1080/15459620801922211

[19] Jones T, Kumar S. Comparison of ergonomic risk assessment output in four sawmill jobs. Int $\mathrm{J}$ Occup Saf Ergon. 2010;16(1):105-111. doi:10.1080/10803548.2010. 11076834

[20] Hansson G-A, Strömberg U, Larsson B, et al. Electromyographic fatigue in neck/shoulder muscles and endurance in women with repetitive work. Ergonomics. 1992;35(11):1341-1352. doi:10.1080/00140139208967397

[21] Sundelin G, Hagberg M. Electromyographic signs of shoulder muscle fatigue in repetitive arm work paced by the methods-time measurement system. Scand J Work Environ Health. 1992;18(4):262-268. doi:10.5271/sjweh.1579

[22] Sundelin G. Patterns of electromyographic shoulder muscle fatigue during MTM-paced repetitive arm work with and without pauses. Int Arch Occup Environ Health. 1993;64(7):485-493. doi:10.1007/BF00381096

[23] Mathiassen SE, Winkel J. Physiological comparison of three interventions in light assembly work: reduced work pace, increased break allowance and shortened working days. Int Arch Occup Environ Health. 1996;68(2):94-108. doi:10.1007/BF00381241

[24] Bosch T, de Looze MP, van Dieën JH. Development of fatigue and discomfort in the upper trapezius muscle during light manual work. Ergonomics. 2007;50:161-177. doi:10.1080/00140130600900282

[25] Luttmann A, Jäger M, Laurig W. Electromyographical indication of muscular fatigue in occupational field studies. Int J Ind Ergon. 2000;25:645-660. doi:10.1016/S0169-8141(99) 00053-0

[26] de Looze M, Bosch T, van Dieën J. Manifestations of shoulder fatigue in prolonged activities involving low-force contractions. Ergonomics. 2009;52(4):428-437. doi:10.1080/00140130802707709

[27] Cifrek M, Medved V, Tonković S, et al. Surface EMG based muscle fatigue evaluation in biomechanics. Clin Biomech (Bristol, Avon). 2009;24(4):327-340. doi:10.1016/j.clinbiomech.2009.01.010

[28] Dimitrova NA, Dimitrov GV. Interpretation of EMG changes with fatigue: facts, pitfalls, and fallacies. J Electromyogr Kinesiol. 2003;13(1):13-36. doi:10.1016/ S1050-6411(02)00083-4 
[29] Merletti R, Farina D, Gazzoni M. The linear electrode array: a useful tool with many applications. J Electromyogr Kinesiol. 2003;13:37-47. doi:10.1016/S1050-6411(02)00082-2

[30] Merletti R, Botter A, Troiano A, et al. Technology and instrumentation for detection and conditioning of the surface electromyographic signal: State of the art. Clin Biomech (Bristol, Avon). 2009;24:122-134. doi:10.1016/j.clinbiomech.2008.08.006

[31] Zwarts MJ, Stegeman DF. Multichannel surface EMG: basic aspects and clinical utility. Muscle Nerve. 2003;28(1):1-17. doi: $10.1002 /$ mus. 10358

[32] Hägg GM, Luttmann A, Jäger M. Methodologies for evaluating electromyographic field data in ergonomics. J Electromyogr Kinesiol. 2000;10(5):301-312. doi:10.1016/ S1050-6411(00)00022-5

[33] Luttmann A, Jäger M, Sökeland J, et al. Electromyographical study on surgeons in urology, part II: determination of muscular fatigue. Ergonomics. 1996;39:298-313. doi:10.1080/00140139608964460

[34] Kumar S, Mital A, editors. Electromyography in ergonomics. London: Taylor and Francis; 1996.

[35] Gazzoni M. Multichannel surface electromyography in ergonomics: potentialities and limits. Hum Factors Man. 2010;20(4):255-271. doi:10.1002/hfm.20219

[36] Farina D, Merletti R. A novel approach for estimating muscle fiber conduction velocity by spatial and temporal filtering of surface EMG signals. IEEE Trans Biomed Eng. 2003;50(12):1340-1351. doi:10.1109/TBME.2003.819847

[37] Farina D, Merletti R. Methods for estimating muscle fibre conduction velocity from surface electromyographic signals. Med Biol Eng Comput. 2004;42(4):432-445. Review. Erratum in: Med Biol Eng Comput. 2004;42(5):732. doi:10.1007/BF02350984

[38] Lindstrom L, Kadefor R, Petersen I. An electromyographic index for localized muscle fatigue. J Appl Physiol. 1997;43(4):750-754.

[39] Farina D, Fortunato E, Merletti R. Noninvasive estimation of motor unit conduction velocity distribution using linear electrode arrays. IEEE Trans Biomed Eng. 2000;47(3):380388. doi: $10.1109 / 10.827303$

[40] Qin J, Lin J-H, Faber GS, et al. Upper extremity kinematic and kinetic adaptations during a fatiguing repetitive task. J Electromyogr Kinesiol. 2014;24(3):404 411. doi:10.1016/j.jelekin.2014.02.001

[41] Roman-Liu D, Tokarski T, Wójcik K. Quantitative assessment of upper limb muscle fatigue depending on the conditions of repetitive task load. J Electromyogr Kinesiol. 2004;14(6):671-682. doi:10.1016/j.jelekin.2004.04.002

[42] Iridiastadi $H$, Nussbaum MA. Muscular fatigue and endurance during intermittent static efforts: effects of contraction level, duty cycle, and cycle time. Hum Factors. 2006;48(4):710-720. doi:10.1518/00187200677916 6389

[43] Iridiastadi H, Nussbaum MA. Muscle fatigue and endurance during repetitive intermittent static efforts: development of prediction models. Ergonomics. 2006;49(4):344-360. doi:10.1080/00140130500475666

[44] Bosch T, de Looze MP, Kingma I, et al. Electromyographical manifestations of muscle fatigue during different levels of simulated light manual assembly work. J Electromyogr Kinesiol. 2009;19:e246-e256. doi:10.1016/j.jelekin.2008.04.014

[45] Buckle P, Devereux J. Work-related neck and upper limb musculoskeletal disorders. Bilbao: European Agency for Safety and Health at Work; 1999.

[46] Rab G, Petuskey K, Bagley A. A method for determination of upper extremity kinematics. Gait Posture. 2002;15:113119. doi:10.1016/S0966-6362(01)00155-2

[47] Wu G, Siegler S, Allard P, et al. ISB recommendation on definitions of joint coordinate system of various joints for the reporting of human joint motion - part I: ankle, hip, and spine. International Society of Biomechanics. J Biomech. 2002;35(4):543-548.

[48] $\mathrm{Wu} \mathrm{G}$, van der Helm FCT, Veeger HEJ, et al. ISB recommendation on definitions of joint coordinate systems of various joints for the reporting of human joint motion part II: shoulder, elbow, wrist and hand. J Biomech. 2005;38(5):981-992. doi:10.1016/j.jbiomech.2004.05.042

[49] Barbero M, Merletti R, Rainoldi A. Atlas of muscle innervation zones: understanding surface electromyography and its applications. New York (NY): Springer; 2012.

[50] Qin J, Lin J-H, Buchholz B, et al. Shoulder muscle fatigue development in young and older female adults during a repetitive manual task. Ergonomics. 2014;57(8): 1201-1212. doi:10.1080/00140139.2014.914576

[51] Merletti R, Parker PJ. Electromyography: physiology, engineering, and non-invasive applications. New York (NY): Wiley-IEEE Press; 2004.

[52] Hermens HJ, Freriks B. European recommendations for surface electromyography: results of the SENIAM Project. Enschede: Roessingh Research and Development; 1999. 